\title{
Conservative Treatment of Huge Fibrous Dysplasia Causing Dysfunction in the Maxilla
}

\author{
Onur Şahin ${ }^{1 *}$ (D), Xhini Rizaj ${ }^{1}$ (D), Toghrul Aliyev ${ }^{1}$ (D) and Ceren Özeren Keşkek ${ }^{2}$ (D) \\ ${ }^{1}$ Faculty of Dentistry, Department of Oral and Maxillofacial Surgery, Izmir Katip Çelebi University, Izmir, Turkey \\ ${ }^{2}$ Faculty of Dentistry, Department of Oral and Maxillofacial Radiology, Izmir Katip Çelebi University, izmir, Turkey
}

\begin{abstract}
Fibrous dysplasia (FD) is a non-malignant condition in which normal bone and marrow are replaced by fibrous tissue and haphazardly distributed woven bone. In the craniomaxillofacial skeleton, the maxilla is more frequently affected by FD than the mandible and generally affects adjacent bones such as the sphenoid, zygomatic and the frontal bones. The aim of this case report is to present the clinical, radiological, histological findings and conservative treatment of patient diagnosed with huge FD caused dysfunction in maxilla.
\end{abstract}

\section{Keywords}

Fibrous dysplasia, Maxilla, Conservative treatment

\section{Introduction}

Fibrous dysplasia (FD) is a skeletal anomaly in which deficiently organized and insufficiently mineralized immature bone and fibrous tissue takes the place of normal bone. FD may touch any bone in the body; nevertheless, the jaw, the skull, the pelvis, the long bones, the ribs are most generally affected [1]. The disease may involve a single bone (monostotic) (characterizing most cases of FD) or multiple bones (polyostotic) [2]. The polyostotic type may be correlated to the McCune-Albright syndrome in which characteristic Caféau-lait spots are combined with skeletal abnormalities and endocrine abnormalities, as well as the Jaffe-Lichtenstein syndrome in which patients demonstrate the same symptoms, excluding endocrine abnormalities $[3,4]$. FD of bone evolves from activating missense mutations in Gs alpha gene in pluripotent embryonic stem cells. The inheritance of these mutations remains in a population of postnatal skeletal stem cells or mesenchymal stem cells which direct the formation of atypical bone in FD [5]. In the craniomaxillofacial skeleton, the maxilla is more frequently affected by FD than the mandible and generally affects adjacent bones such as the sphenoid, zygomatic, and the frontal bones [6]. The major clinical symptom of FD is painless swelling in the affected bones. The overgrowth of the bone can cause facial asymmetry, deformation, malocclusion caused by teeth migration and exophthalmos related to the anatomical sites and structures implicated [7]. Radiographic characteristics of FD differ from radiolucent (initial stages) to radiopaque (mature) lesions, in order to the combination of fibrous and osseous elements. The affected bone presents expansion and a ground-glass appear- ance is generally reported in progressive stages [8]. Histological examination is essential for definitive diagnosis. The aim of this case report is to present the clinical, radiological and histological findings of patients diagnosed with FD in maxilla. Taking into consideration the gender, age, recurrence probability, size of lesion and involvement of adjacent anatomic structures, the ability to define the accurate diagnosis and plan the most appropriate treatment by the clinician is aimed.

\section{Case Presentation}

A systemically healthy, 41-year-old woman has referred to our clinic with a complaint of asymmetry and swelling in maxillary left posterior region for the past 2 years. A biopsy had been performed 2 years before the visit with clear diagnosis of FD. On the clinical examination, teeth migration, malocclusion and asymmetry were obviously visible. There was no swelling present elsewhere in the body, and café-au-lait spots were absent. Routine investigations such as hemogram,

*Corresponding author: Assit. Prof. Dr. Onur Sahin, Faculty of Dentistry, Department of Oral and Maxillofacial Surgery, İzmir Katip Çelebi Universty, Aydınlıkevler Mah. 6782 Sok. No: 4835640 Çiğli, İzmir, Turkey, Tel: 00905054410192, Fax: +90(232)-325-25-35

Accepted: October 17, 2020

Published online: October 19, 2020

Citation: Şahin O, Rizaj X, Aliyev T, et al. (2020) Conservative Treatment of Huge Fibrous Dysplasia Causing Dysfunction in the Maxilla. J Oral Healthc 1(1):7-10 


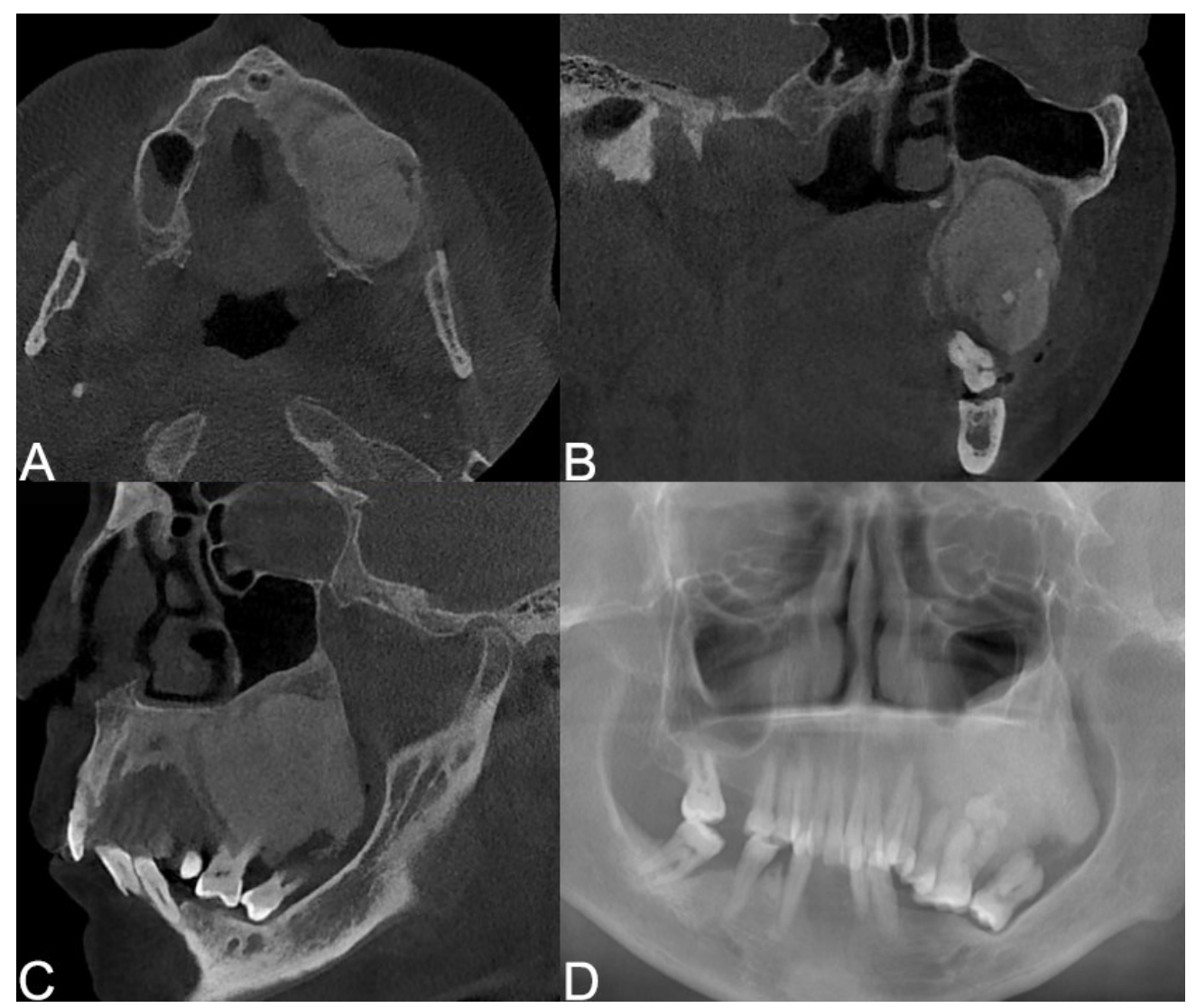

Figure 1: (A) Axial view showing the extension of the lesion mediolaterally; (B) Coronal view of the lesion; (C) Sagittal view showing the extension of the lesion anteroposteriorly and superior-inferiorly; (D) Panoramic radiography of the lesion on the left upper jaw.

serum calcium and serum alkaline phosphatase (ALP) were performed. All parameters were within normal limits except ALP. In the orthopantomography (OPG) and cone beam computed tomography (CBCT) examination, a ground glass image consistent with the characteristic appearance of fibrous dysplasia was located in the inferior part of the left maxillary sinus (Figure 1). It showed an expansile mass lesion at the left maxillary region measuring about $6.8 \times 7.4 \times 6 \mathrm{~cm}$. In this case, it was decided to extract the teeth and remove the large bone fragment causing dysfunction for a biopsy sample (Figure 2A, Figure 2B and Figure 2C). Since the patient didn't prefer general anesthesia, hemimaxilectomy was not the first selection. So, we preferred a conservative treatment involving ultrasonic piezoelectric bone surgery and osteotomy. A large part of the lesion was excised as the huge expansion caused dysfunction. Bone deformities and asymmetry were corrected. No treatment of other bones was done because there were no ocular symptoms and patient was unwilling too. Histopathological examination of the incisional biopsy revealed irregular shaped compact bone lamellae in the loose fibrous stroma and was diagnosed as fibrous dysplasia (Figure 2D). Since FD may have genetic inheritance, 2 children and 1 brother of the patient were examined clinically and radiologically. However, no signs of fibrous dysplasia were encountered. The patient was followed-up for two-year and the routine follow-up was continued. No infective complications and expansion occurred at two-year follow-up.

\section{Discussion}

FD is a non-malignant condition in which normal bone and marrow are replaced by fibrous tissue and haphazardly distributed woven bone [1]. All forms of fibrous dysplasia are seen equally in men and women without gender inequity. However, Kruse, et al. [9] reported that the disease was more common in women. It is often thought that lesions will stabilize with the completion of skeletal development in the first three decades of life. However, in this case the age of patients is over 40 years.

The FD has three radiological patterns as follows; the first type is pagetoid with bone expansion and alternate areas of radiodensity and radiolucency. The second pattern is sclerotic, with bone expansion and a homogenous radiodensity (a ground glass appearance). The third type is cyst-like, usually a round or oval lesion with a sclerotic border [10]. Our case matches with the first type of Fries' descriptions.

MacDonald Jankowski [11] reported that tooth displacement might also occur and lamina dura could be absent in FD patients. Petrikowski, et al. [12] suggested that the loss of lamina dura could be used as an ancillary diagnostic feature for FD. In the present cases, the absence of lamina dura was observed and tooth displacement was present.

Regarding the treatment of fibrous dysplasia in literature there are various conservative and invasive methods [13]. Conservative treatments such as debulking, contour and 


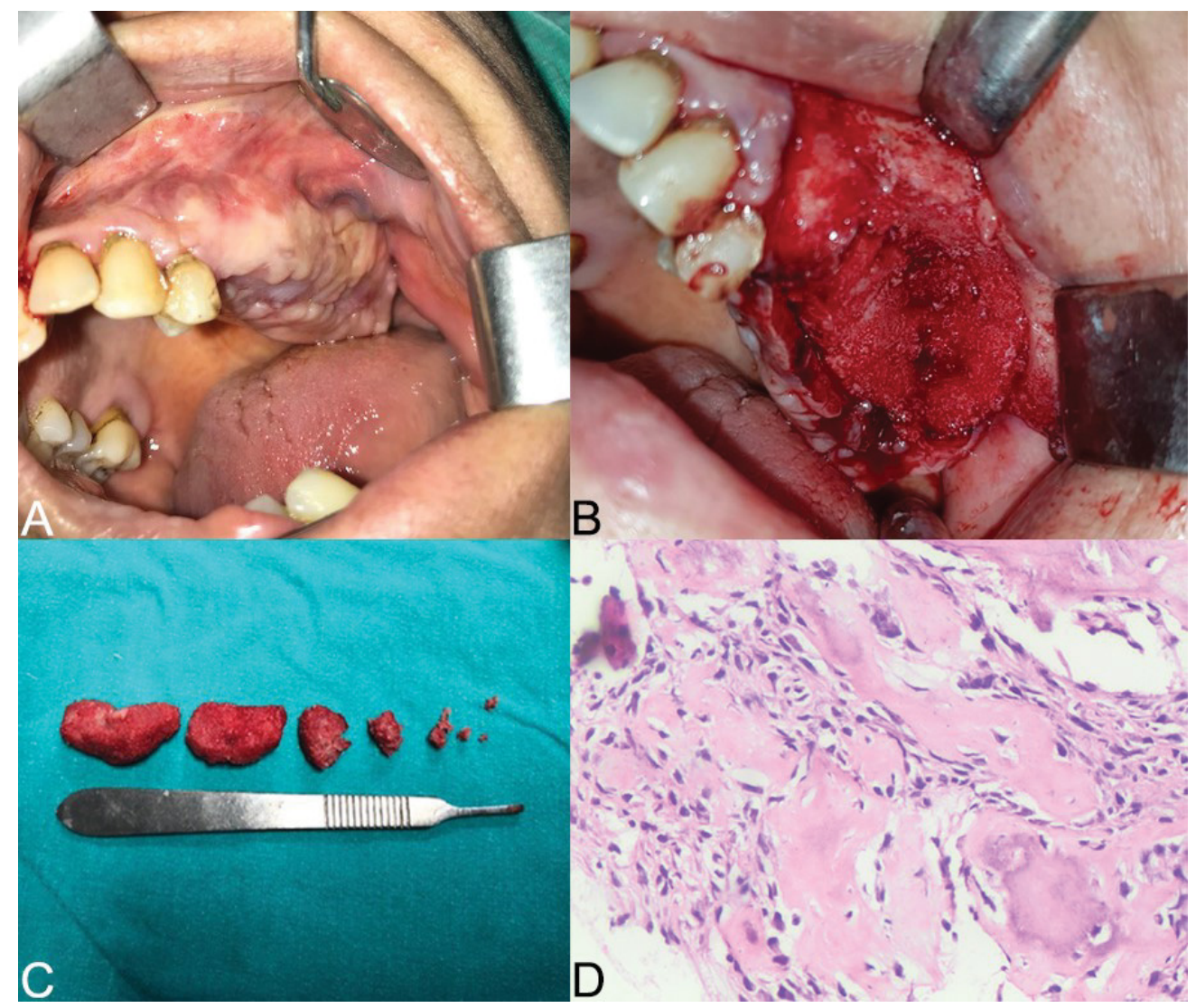

Figure 2: (A) Intraoral view of the lesion in the left posterior maxilla region after extraction; (B) Intraoperative view of the lesion; (C) Lesion removed by ultrasonic piezo electric bone surgery under the local anesthesia; (D) Uniformly distributed C shaped or Chinese figure-like trabeculae of woven/immature bone within proliferating fibroblastic and vascularized stroma.

shaving can be applied for extreme lesions. However, especially in the $2^{\text {nd }}$ and $3^{\text {rd }}$ decades of life, regrowth may occur [14]. Differential diagnosis of osteogenic sarcoma or osteomyelitis such as biopsy is recommended for extensive lesions before surgery to rule out secondary lesions. Lesions that are refractory to debulking, small monostotic lesions, and aggressive lesions may require complete surgical removal. While radiotherapy is controversial in treating the disease because it increases the risk of sarcomatous transformation $[13,14]$.

In craniomaxillofacial fibrous dysplasia surgical treatment is not advisable if there are no severe deformations in the craniomaxillofacial bones. Also if the lesions are asymptomatic and there is no risk of fractures every 6 months clinical and radiological follow-up examinations are advised.

In this present case, the patient didn't have pain, diplopia or visual impairment. The main concern was the apparent asymmetry on the side of the lesion and due to changes in occlusion reduction of bite and dilatative abilities was present. The patient underwent non-invasive surgical operation using piezosurgery. The loss of chewing function due to the extrusion of the teeth in the relevant area was resolved by extraction of the teeth and partial removal of the lesion.

\section{Conclusion}

As a result, it should be remembered that fibrous dysplasia, which is frequently seen in young ages, may also occur in older ages. The patient should be relieved that the lesion is not malignant. Patient controls should not be neglected in case of recurrence and malignant transformation risk, even though it is rare.

\section{Acknowledgement}

None.

\section{Conflicts of Interest}

There are no conflicts of interest to declare.

\section{Consent}

Consent for publication was taken from the patient.

\section{Authors Declaration}

The manuscript has not been published and is not under consideration for publication in any other journal. Instructions to the author were read. We accept all conditions and publication rights. All authors have approved the manuscript and its submission to the journal. We have no funding sources to declare.

\section{References}

1. Şahin O, Odabaşı O (2018) Clinical and histopathological evaluation of odontogenic cysts according to World Health Organization new classification of odontogenic cysts: A preliminary study. Ponte Int J Sci Res 74: 59-74. 
2. MacDonald DS (2015) Maxillofacial fibro-osseous lesions. Clin Radiol 70: 25-36.

3. Albright F, Butler AM, Hampton AO, et al. (1937) Syndrome characterized by osteitis fibrosa disseminata, areas of pigmentation and endocrine dysfunction, with precocious puberty in females - report of five cases. N Engl J Med 216: 727-746.

4. Lichtenstein L, Jaffe HL (1942) Fibrous dysplasia of bone. A condition affecting one, several or many bones, the graver cases of which may present abnormal pigmentation of skin, premature sexual development, hyperthyroidism or still other extra skeletal abnormalities. Arch Pathol 33: 777-816.

5. Chandavarkar V, Patil PM, Bhargava D, et al. (2018) A rare case report of craniofacial fibrous dysplasia. J Oral Maxillofac Pathol 22: 406-409.

6. McCarthy EF (2013) Fibro-osseous lesions of the maxillofacial bones. Head Neck Pathol 7: 5-10.

7. Mainville GN, Turgeon DP, Kauzman A (2017) Diagnosis and management of benign fibro-osseous lesions of the jaws: A current review for the dental clinician. Oral Dis 23: 440-450.

8. Gupta D, Garg P, Mittal A (2017) Computed tomography in craniofacial fibrous dysplasia: A case series with review of literature and classification update. Open Dent J 11: 384-403.
9. Kruse A, Pieles U, Riener MO, et al. (2009) Craniomaxillofacial fibrous dysplasia: A 10-year database 1996-2006. Br J Oral Maxillofac Surg 47: 302-305.

10. Fries JW (1957) The roentgen features of fibrous dysplasia of the skull and facial bones; a critical analysis of thirty-nine pathologically proved cases. Am J Roentgenol Radium Ther Nucl Med 77: 71-88.

11. MacDonald-Jankowski D (2009) Fibrous dysplasia: A systematic review. Dentomaxillofac Radiol 38: 196-215.

12. Petrikowski CG, Pharoah MJ, Lee L, et al. (1995) Radiographic differentiation of osteogenic sarcoma, osteomyelitis, and fibrous dysplasia of the jaws. Oral Surg Oral Med Oral Pathol Oral Radiol 80: 744-750.

13. Javaid MK, Boyce A, Appelman-Dijkstra N, et al. (2019) Best practice management guidelines for fibrous dysplasia/McCune-Albright syndrome: A consensus statement from the FD/MAS international consortium. Orphanet J Rare Dis 14: 139.

14. Davidova LA, Bhattacharyya I, Islam MN, et al. (2020) An analysis of clinical and histopathologic features of fibrous dysplasia of the jaws: A series of 40 cases and review of literature. Head Neck Pathol 14: 353-361.

DOI: $10.36959 / 704 / 674$

Copyright: (c) 2020 Şahin O, et al. This is an open-access article distributed under the terms of the Creative Commons Attribution License, which permits unrestricted use, distribution, and reproduction in any medium, provided the original author and source are credited. 